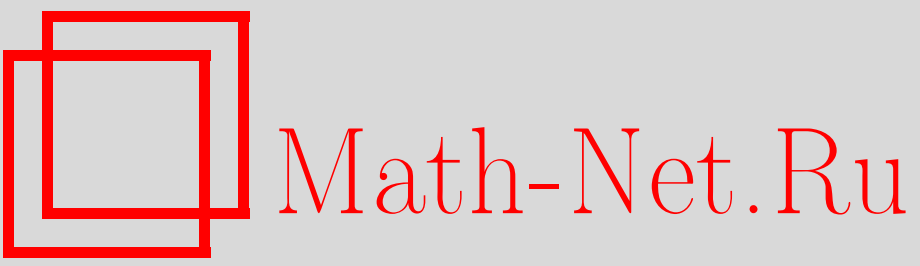

М. И. Илолов, Дробные линейные интегро-дифференциальные уравнения Вольтерра в банаховых пространствах, Итоги науки и техн. Сер. Соврем. мат. и ее прил. Темат. обз., 2019, том 173, 58-64

DOI: https://doi.org/10.36535/0233-6723-2019-173-58-64

Использование Общероссийского математического портала Math-Net.Ru подразумевает, что вы прочитали и согласны с пользовательским соглашением

http://www.mathnet.ru/rus/agreement

Параметры загрузки:

IP: 34.227 .88 .159

26 апреля 2023 г., $13: 11: 10$ 


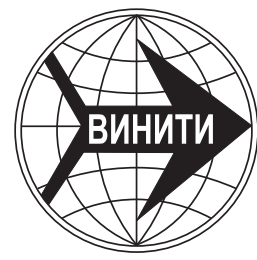

ИТОГИ НАУКИ И ТЕХНИКИ.

Современная математика и ее приложения.

Тематические обзоры.

Том 173 (2019). C. 58-64

DOI: $10.36535 / 0233-6723-2019-173-58-64$

УДК 517.983.5, 517.968.7

\title{
ДРОБНЫЕ ЛИНЕЙНЫЕ ИНТЕГРО-ДИФФЕРЕНЦИАЛЬНЫЕ УРАВНЕНИЯ ВОЛЬТЕРРА В БАНАХОВЫХ ПРОСТРАНСТВАХ
}

(c) 2019 г. $\quad$ М. И. ИЛОЛОВ

\begin{abstract}
АннотАция. В работе представлены основы теории дробных линейных интегро-дифференциальных уравнений Вольтерра типа свертки в банаховых пространствах. Установлено, что существование дробного резольвентного оператора для таких уравнений равносильно корректности постановки начальной задачи для них. В рамках этого подхода доказана теорема типа Хилле-Иосида.

Ключевые слова: дробная производная Капуто, дробная резольвента, интегро-дифференциальное уравнение Вольтерра, функция Миттаг-Леффлера, теорема Хилле-Иосида, дробное резольвентное уравнение.
\end{abstract}

\section{FRACTIONAL LINEAR VOLTERRA INTEGRO-DIFFERENTIAL EQUATIONS IN BANACH SPACES}

\author{
(c) 2019 M. I. ILOLOV
}

\begin{abstract}
The paper presents the foundations of the theory of linear fractional Volterra integrodifferential equations of convolution type in Banach spaces. It is established that the existence of a fractional resolvent operator for such equations is equivalent to the well-posedness of the formulation of the initial problem for them. Within the framework of this approach, a theorem of the Hille-Yosida type is proved.
\end{abstract}

Keywords and phrases: Caputo fractional derivative, fractional resolvent, Volterra integrodifferential equation, Mittag-Leffler function, Hille-Yosida theorem, fractional resolvent equation.

AMS Subject Classification: 45D05, 34A08

1. Введение. Аппарат дробных интегро-дифференциальных уравнений в частных производных вполне адекватно описывает эволюцию фрактальных физических явлений и процессов, позволяя учитывать эредитарные свойства типа гистерезиса, усредненно влияющие на текущее состояние системы.

Интегро-дифференциальные уравнения типа Вольтерра рассматривались в $[5,8,9]$ в качестве интерполяции между моделями обычного потока тепла (параболическая задача) и потока тепла Гуртина-Пипкина (гиперболическая задача). Позднее в [3] рассматривалась иная интерполяционная ситуация, когда начально-краевая задача для уравнения Вольтерра является моделью теории упругости.

В [9] изучались продольные движения упругой балки равномерного поперечного сечения длины $l$ с закрепленными концами. Соответствующая граничная задача для динамического уравнения имеет вид

$$
u_{t t}(x, t)=\sigma_{x}(x, t)+b(x, t), u(0, t)=u(l, t)=0, \quad 0<x<l, \quad t>0,
$$


где $u$-перемещение, $\sigma$ - напряжение и $b$ - внешние силы. Предполагается, что для $t \leqslant 0$ балка находится в состоянии покоя. Соотношения, связывающие напряжения $\sigma(x, t)$ и деформации $u_{x}(x, t)$, являются предметом анализа различных теорий. В линейной теории вязкоупругости определяющим соотношением является уравнение

$$
\sigma(x, t)=b_{1} u_{x}(x, t)-\int_{0}^{t} a(t-\tau) u_{x}(x, \tau) d \tau
$$

которое отражает свойство памяти. Здесь $b_{1}-$ положительная постоянная и $a-$ положительная невозрастающая функция.

В физике твердого тела таким соотношением является уравнение Кельвина-Фойгта

$$
\sigma(x, t)=d u_{x}(x, t)+c u_{x t}(x, t)
$$

где $d, c$ - положительные постоянные.

Подставляя (2) и (3) в уравнение (1), получаем эволюционное уравнение, причем в случае (2) имеем конечную скорость распространения, а в случае (3) - бесконечную. В обоих случаях решения задачи (1) асимптотически устойчиво.

Интерполяционная модель задается формулой

$$
\sigma(x, t)=e u_{x}(x, t)+\frac{1}{\Gamma(1-\alpha)} \frac{\partial}{\partial t} \int_{0}^{t}(t-\tau)^{-\alpha} b(t-\tau) u_{x}(x, \tau) d \tau, \quad 0<\alpha<1
$$

где $e>0, \Gamma$ - гамма-функция Эйлера.

В случае $\alpha \rightarrow 0$ формула (4) превращается в (2) с $b_{1}=l+b(0)$ и $a(t)=-b(t)$, а в случае $\alpha \rightarrow 1-\mathrm{в}(3)$ с $d=e$ и $c=b(0)$.

Подстановка (4) в (1) дает задачу с бесконечной скоростью распространения и асимптотически устойчивым решением, если выполнены соответствующие предположения относительно $b$.

В [9] дана абстрактная формулировка соответствующей задачи для ограниченных операторов в гильбертовом пространстве.

В наиболее общей постановке задача типа (4) можно исследовать путем сведения к дробным интегро-дифференциальным уравнениям в банаховом пространстве.

В настоящей работе рассматривается начальная абстрактная задача вида

$$
{ }^{c} D_{t}^{\alpha} u(t)=A u(t)+\int_{0}^{t} B(t-s) u(s) d s+f(t), \quad u(0)=u_{0},
$$

где ${ }^{c} D_{t}^{\alpha}$ - дробный дифференциальный оператор Капуто, $0<\alpha<1, A-$ замкнутый линейный оператор, плотно определенный в банаховом пространстве $X,\{B(t)\}_{t \geqslant 0}$ - такое семейство линейных замкнутых операторов, что $D(B(t)) \supset D(A)$ для всех $t \geqslant 0$, функции $B(t) x$ сильно измеримы по $x \in D(A)$. Соответствующая начальная задача для интегро-дифференциальных уравнений целых порядков подробно изучена в $[1,6,10]$. В $[2,7]$ рассматриваются различные частные случаи интегро-дифференциальных уравнений дробного порядка.

Интерес к задачам типа (5) обусловлен их широким применением в различных областях физики, химии, биологии и др. Например, как установлено в [6], дробные дифференциальные и интегро-дифференциальные уравнения описывают аномальную диффузию для различных фрактальных объектов дробной размерности (аморфные полупроводники, сильно пористые неоднородные материалы, космические лучи сверхвысокой энергии) с учетом памяти и других наследственных свойств. Известны также приложения в электрохимии, реологии и популяционной динамике.

2. Предварительные сведения. В этом разделе вводим некоторые определения, обозначения и предварительные факты, необходимые в дальнейшем. Пусть $\alpha>0$ и $m=[\alpha]$ означает наименьшее целое число, большее или равное $\alpha$. Пусть $X$-банахово пространство, $\mathbb{R}_{+}=[0, \infty)$. Через 
$L^{1}((0, T): X)$ обозначим пространство всех интегрируемых по Бохнеру функций $u:(0, T) \rightarrow X$, которое является банаховым пространством с нормой

$$
\|u\|_{1}=\int_{0}^{T}\|u(t)\| d t
$$

Обозначим через $C([0, T] ; X)$ (соответственно, $\left.C^{1}([0, T] ; X)\right)$ банаховы пространства непрерывных (соответственно, непрерывно дифференцируемых) функций $u:[0, T] \rightarrow X\left(u^{\prime}:[0, T] \rightarrow X\right)$ с нормами

$$
\|u\|_{C}=\sup _{t \in[0, T]}\|u(t)\|_{X}, \quad\|u\|_{C^{1}}=\sup _{t \in[0, T]}\left(\|u(t)\|_{X}+\left\|u^{\prime}(t)\right\|_{X}\right) .
$$

Обозначим через $\rho(A)$ резольвентное множество оператора $A$, т.е. множество всех комплексных чисел $\lambda$, для которых $(\lambda I-A)^{-1}$ является линейным ограниченным оператором на $X$. Оператор $R(\lambda, A)=(\lambda I-A)^{-1}$ называется резольвентным оператором оператора $A$. Введем обозначение $\mathbb{N}_{0}=\mathbb{N} \cup\{0\}$, где $\mathbb{N}$ - множество натуральных чисел. Для свертки двух функций $f$ и $g$ используется сокращение

$$
(f * q)(t)=\int_{0}^{t} f(t-\tau) g(\tau) d \tau .
$$

Через $I$ будем обозначать один из промежутков $(0, T)$ или $[0, T]$ либо $\mathbb{R}_{+}$. Пусть $m \in \mathbb{N}, 1 \leqslant p<\infty$. Пространство Соболева определяется следующим образом:

$$
W^{m, p}(I, X)=\left\{u \mid \exists \varphi \in L^{1}(I, X): u(t)=\sum_{k=0}^{m-1} c_{k} \frac{t^{K}}{k !}+\frac{t^{m-1}}{(m-1) !} * \varphi(t), t \in I\right\} .
$$

Заметим, что здесь $\varphi(t)=u^{m}(t), c_{k}=u^{(k)}(0)$.

Определение 1. Дробный интеграл Римана-Лиувилля порядка $\alpha$ определяется равенством

$$
J_{t}^{\alpha} u(t)=\frac{1}{\Gamma(\alpha)} \int_{0}^{t}(t-\tau)^{\alpha-1} u(\tau) d \tau, \quad u \in L^{1}((0, T) ; X),
$$

где $\Gamma(\alpha)$ - гамма-функция Эйлера.

Интеграл Римана-Лиувилля (6) может быть записан в виде

$$
J_{t}^{\alpha} u(t)=\left(g_{\alpha} * u\right)(t)
$$

где

$$
g_{\alpha}= \begin{cases}\frac{t^{\alpha-1}}{\Gamma(\alpha)}, & t>0 \\ 0, & t \leqslant 0\end{cases}
$$

Полагая $J_{t}^{0} u(t)=u(t)$ и учитывая $(7)$, получим полугрупповое свойство для $J_{t}^{\alpha}$ :

$$
J_{t}^{\alpha} J_{t}^{\beta}=J_{t}^{\alpha+\beta}, \quad \alpha, \beta \geqslant 0 .
$$

Определение 2. Дробная производная Римана-Лиувилля порядка $\alpha$ определяется равенством

$$
D_{t}^{\alpha} u(t)=\frac{1}{\Gamma(m-\alpha)} \frac{d^{m}}{d t^{m}} \int_{0}^{t}(t-\tau)^{m-\alpha-1} u(\tau) d \tau
$$

где $u \in L^{1}((0, T) ; X), g_{m-\alpha} * u \in W^{m, 1}((0, T) ; X)$. Если $\alpha=m, m \in \mathbb{N}$, то $D_{t}^{m} \equiv d^{m} / d t^{m}$. 
Дифференциальный оператор Римана-Лиувилла $D_{t}^{\alpha}$ является левым обратным для интегрального оператора $J_{t}^{\alpha}$, однако в общем случае не является правым обратным, так что

$$
\begin{gathered}
D_{t}^{\alpha} J_{t}^{\alpha} u=u, \quad u \in L^{1}((0, T) ; X), \\
\left(J_{t}^{\alpha} D_{t}^{\alpha} u\right)(t)=u(t)-\sum_{k=0}^{\infty}\left(g_{m-\alpha} * u\right)^{k}(0) g_{\alpha+k+1-m}(t),
\end{gathered}
$$

где $u \in L^{1}((0, T) ; X), g_{m-\alpha} * a \in W^{m, 1}((0, T) ; X)$.

Определение 3. Дробная производная Капуто порядка $\alpha$ определяется равенством

$$
{ }^{C} D_{t}^{\alpha} u(t)=D_{t}^{\alpha}\left[u(t)-\sum_{k=0}^{\infty} u^{(k)}(0) g_{k+1}(t)\right],
$$

где $u \in L^{1}((0, T) ; X) \cap C^{m-1}((0, T) ; X), g_{m-\alpha} * u \in W^{m, 1}((0, T) ; X)$.

Дифференциальный оператор Капуто также является левым обратным для интегрального оператора $J_{t}^{\alpha}$, однако в общем случае не является правым обратным, так что

$$
\begin{gathered}
{ }^{C} D_{t}^{\alpha} J_{t}^{\alpha} u=u, \quad u \in L^{1}((0, T) ; X), \\
\left(J_{t}^{\alpha} D_{t}^{\alpha} u\right)(t)=u(t)-\sum_{k=0}^{m-1} u^{(k)}(0) g_{k+1}(t),
\end{gathered}
$$

где $u \in L^{1}((0, T) ; X) \cap C^{m-1}((0, T) ; X), g_{m-\alpha} * u \in W^{m, 1}((0, T) ; X)$.

В равенствах (6), (9) и (12) интегралы понимаются в смысле Бохнера.

Определим преобразование Лапласа для дробной производной Капуто в виде

$$
L\left({ }^{C} D_{t}^{\alpha}\right)(\lambda)=\lambda^{\alpha} \hat{u}(\lambda)-\sum_{k=0}^{m-1} u^{(k)}(0) \lambda^{\alpha-1-k},
$$

где $\hat{u}(\lambda)$ - преобразование Лапласа функции $u(t)$, т.е.

$$
\hat{u}(\lambda)=\int_{0}^{\infty} e^{-\lambda t} u(t) d t, \quad \operatorname{Re} \lambda>\omega,
$$

где $\operatorname{Re} \lambda$ - действительная часть комплексного числа $\lambda$. Наиболее полезными в дальнейшем являются следующие случаи:

$$
\begin{aligned}
& \text { (1) } \quad 0<\alpha \leqslant 1, \quad L\left({ }^{C} D_{t}^{\alpha} u\right)(\lambda)=\lambda^{\alpha} \hat{u}(\lambda)-\lambda^{\alpha-1} u(0) ; \\
& \text { (2) } 1<\alpha \leqslant 2, \quad L\left({ }^{C} D_{t}^{\alpha} u\right)(\lambda)=\lambda^{\alpha} \hat{u}(\lambda)-\lambda^{\alpha-1} u(0)-\lambda^{\alpha-2} u(0) .
\end{aligned}
$$

Определение 4. Функция определенная с помощью равенства

$$
E_{\alpha, \beta}(z)=\sum_{k=0}^{\infty} \frac{z^{k}}{\Gamma(\alpha k+\beta)}, \quad \alpha, \beta>0, \quad z \in \mathbb{C},
$$

называется функиией Миттаг-Леффлера.

Функция Миттаг-Леффлера связана с интегралом Лапласа следующим образом:

$$
\int_{0}^{\infty} e^{-\lambda t} t^{\beta-1} E_{\alpha, \beta}\left(\omega t^{\alpha}\right) d t=\frac{\lambda^{\alpha-\beta}}{\lambda^{\alpha-\omega}}, \quad \operatorname{Re} \lambda>\omega^{1 / \alpha}, \quad \omega>0 .
$$

Для этой функции имеют место следующие асимптотические представления:

$$
\begin{gathered}
E_{\alpha, \beta}=\frac{1}{\alpha} z^{1-\beta / \alpha} \exp \left(z^{1 / \alpha}\right)+e_{\alpha, \beta}(z), \quad|\arg z| \leqslant \frac{\alpha \pi}{2}, \\
E_{\alpha, \beta}(t)=e_{\alpha, \beta}(z), \quad|\arg (-z)|<\left(1-\frac{\alpha}{2}\right) \pi,
\end{gathered}
$$


где

$$
\varepsilon_{\alpha, \beta}(z)=-\sum_{n=0}^{N-1} \frac{z^{-n}}{\Gamma(\alpha-n)}+O\left(|z|^{-N}\right), \quad|z| \rightarrow \infty .
$$

Для операторной функции Миттаг-Леффлера справедливо следующее утверждение.

Лемма 1. Пусть $A \in B(X)$. Тогда для любых $\alpha>0, \beta>0$ справедливо равенство

$$
\mathcal{L}\left[t^{\beta} E_{\alpha, \beta}\left(A t^{\alpha}\right)\right]=\lambda^{\alpha-\beta}\left(\lambda^{\alpha}-A\right)^{-1}
$$

если $R_{e \lambda}>\|A\|^{1 / \alpha}$.

Доказательство. Для $R_{e \lambda}>\|A\|^{1 / \alpha}$ имеем

$$
\sum_{k=0}^{\infty} A^{K} \lambda^{-(k+1)}=\left(\lambda^{\alpha}-A\right)^{-1} .
$$

Тогда

$$
\begin{aligned}
\mathcal{L}\left[t^{\beta-1} E_{\alpha, \beta}\left(A t^{\alpha}\right)\right]=\mathcal{L}\left[t^{\beta-1}\right. & \left.\sum_{k=0}^{\infty} \frac{\left(A t^{\alpha}\right)^{k}}{\Gamma(\alpha k+\beta)}\right]= \\
& =\sum_{k=0}^{\infty} \frac{A^{K} \mathcal{L}\left[t^{\alpha k+\beta-1}\right]}{\Gamma(\alpha k+\beta)}=\lambda^{\alpha-\beta} \sum_{k=0}^{\infty} A^{K} \lambda^{-(k+1) \alpha}=\lambda^{\alpha-\beta}\left(\lambda^{\alpha}-A\right)^{-1} .
\end{aligned}
$$

Отметим также следующие свойства функции Миттаг-Леффлера:

(E1) Пусть $E_{\alpha_{1}}(z)=E_{\alpha}(z)=\sum_{n=0}^{\infty} \frac{z^{n}}{\Gamma(\alpha n+1)}, \alpha>0$. Тогда

$$
\frac{d E_{\alpha}(z)}{d z}=\alpha^{-1} E_{\alpha, \alpha}(z)
$$

(E2) Для $0<\alpha<1$ и любого действительного числа $a$ справедливо равенство

$$
\begin{aligned}
\int_{0}^{t+s} \frac{E_{\alpha}\left(a \tau^{\alpha}\right) d \tau}{(t+s+\tau)^{\alpha}}-\int_{0}^{t} \frac{E_{\alpha}\left(a \tau^{\alpha}\right) d \tau}{(t+s+\tau)^{\alpha}}-\int_{0}^{s} \frac{E_{\alpha}\left(a \tau^{\alpha}\right) d \tau}{(t+s+\tau)^{\alpha}} & \\
& =\alpha \int_{0}^{t} \int_{0}^{t} \frac{E_{\alpha}\left(a \tau_{1}^{\alpha}\right) E_{\alpha}\left(a \tau_{2}^{\alpha}\right) d \tau_{1} d \tau_{1}}{\left(t+s+\tau_{1}+\tau_{2}\right)^{\alpha}}, \quad t, s \geqslant 0 .
\end{aligned}
$$

Заметим, что экспоненциальная функция $E_{1,1}(z)=e^{z}$ обладает полугрупповым свойством $e^{z+v}=e^{z} \cdot e^{v}$. Функция $E_{\alpha}(z)$ не обладает этим свойством. Равенство (15) является характеристическим свойством функции Миттаг-Леффлера в связи с разрешимостью дробного дифференциального уравнения.

3. Основные результаты. Пусть $X$-банахово пространство с нормой $\|\cdot\|$. Рассмотрим вновь начальную задачу

$$
{ }^{c} D_{t}^{\alpha} U(t)=A u(t)+\int_{0}^{t} B(t-s) u(s) d s+f(t), \quad u(0)=U_{0},
$$

где ${ }^{c} D_{t}^{\alpha} U(t)$ - дробная производная Капуто, $0<\alpha<1, A$ - замкнутый линейный оператор плотно определенный в $X,\{B(t)\}_{t \geqslant 0}$ - семейство таких линейных замкнутых операторов, что $D(B(t)) \supset D(A)$ для всех $t \geqslant 0$, функции $B(t) x$ сильно измеримы для $x \in D(A)$ и существует такая скалярная функция из $L_{\mathrm{loc}}^{1}\left(\mathbb{R}_{+}\right)$, что

$$
\|B(t) x\| \leqslant b(t)(\|x\|+\|A x\|)
$$


для всех $x \in D(A)$, почти всех $t \geqslant 0, f \in C\left(\mathbb{R}_{+}, X\right)$ и $u_{0} \in X$.

Определение 5. Семейство линейных ограниченных операторов $\left\{S_{\alpha}(t)\right\}_{t} \geqslant 0,0<\alpha<1$, называется дробным резолъвентным оператором для (16), если выполнены следующие условия:

(S1) $S_{\alpha}(t) x \in C\left(\mathbb{R}_{+}, X\right)$, для всех $x \in X, S_{\alpha}(0)=I$;

(S2) $S_{\alpha}(t) D(A) \subset D(A)$ для всех $t \geqslant 0$ для $x \in D(A), A S_{\alpha}(t) x$ непрерывна и $S(t) x$ непрерывно дифференцируема на $\mathbb{R}_{+}$;

(S3) для всех $x \in D(A)$ и $t \geqslant 0$ выполняются дробные резольвентные уравнения

$$
\begin{aligned}
& { }^{c} D_{t}^{\alpha} S_{\alpha}(t) x=A S_{\alpha}(t) x+\int_{0}^{t} B(t-s) S_{\alpha}(s) x d s, \\
& { }^{c} D_{t}^{\alpha} S_{\alpha}(t) x=S_{\alpha}(t) A x+\int_{0}^{t} S_{\alpha}(t-s) B(s) x d s ;
\end{aligned}
$$

(S4) имеет место равенство

$$
\int_{0}^{t+s} \frac{S_{\alpha}(\tau) x d \tau}{(t+s-\tau)^{\alpha}}-\int_{0}^{t} \frac{S_{\alpha}(\tau) x d \tau}{(t+s-\tau)^{\alpha}}-\int_{0}^{S} \frac{S_{\alpha}(\tau) x d \tau}{(t+s-\tau)^{\alpha}}=\alpha \int_{0}^{t} \int_{0}^{S} \frac{S_{\alpha}\left(\tau_{1}\right) S_{\alpha}\left(\tau_{2}\right)_{d} \tau_{1} d \tau_{2}}{\left(t+s-\tau_{1}-\tau_{2}\right)^{1+\alpha}},
$$

где интегралы определены в сильной операторной топологии.

Имеют место следующие утверждения.

Теорема 1. Существует не более одного дробного резольвентного оператора для (16).

Теорема 2. Пусть (16) допускает дробный резольвентный оператор $S_{\alpha}(t)$ и пусть $u_{0} \in X u$ $f \in C(J, X)$, где $J=[0, a]$. Если и(t) является решением задачи (16) на $J$, то

$$
u(t)=S_{\alpha}(t) U_{0}+\int_{0}^{t} S_{\alpha}(t-\tau) f(s) d s
$$

$\partial л я в$ всех $t \in J$.

Решение $u(t)$ интегрального уравнения (20) называется обобщенным решением задачи (16).

Из теоремы 1 вытекает следующее утверждение.

Следствие 1. Пусть (16) допускает дробный резольвентный оператор. Тогда задача (16) корректно поставлена в обобщенном смысле.

Возникает вопрос о том, для каких начальных значений $u_{0}$ и функций $f$ обобщенное решение является решением задачи (16).

Теорема 3. Предположим, что $S_{\alpha}(t)$ - дробный резольвентный оператор задачи (16). Пусть $u_{0} \in D(A)$ и $A \in C(Y, X) \cap L^{2}(J, X)$ или $f \in W^{1,1}(J, X)$. Тогда решение и $(t)$ уравнения (20) является решением задачи (16).

Следующий результат содержит утверждение о существовании дробного резольвентного оператора для (16) и корректности однородного уравнения

$$
{ }^{c} D_{t}^{\alpha} u(t)=A u(t)+\int_{0}^{t} B(t-s) u(s) d s
$$

в смысле С. Г. Крейна (см. [4]).

Теорема 4. Задача (16) допускает дробный резольвентный оператор $S_{\alpha}(t)$ тогда и только тогда, когда уравнение (21) корректно поставлено. 


\section{СПИСОК ЛИТЕРАТУРЫ}

1. Власов В. В., Ву Джс., Кабирова Г. Р. Корректная разрешимость и спектральные свойства абстрактных гиперболических уравнений с последействием// Совр. мат. Фундам. направл. — 2010. - 35. C. 44-59.

2. Илолов M., Кучакшоев $X$. С. Об абстрактных уравнений с неограниченными нелинейностями и их приложений// Докл. РАН. - 2009. - 428, № 3. - С. 310-312.

3. Илолов М., Кучакшоев Х. С., Гулджонов Д. Н. О дробных линейных уравнениях в банаховых пространствах// Докл. АН Респ. Таджикистан. - 2018. - 61, № 2. - С. 113-119.

4. Крейн C. Г. Линейные дифференциальные уравнения в банаховом пространстве. - М.: Наука, 1967.

5. Da Prato G., Iannelli M. Linear integro-differential equations in Banach spaces// Rend. Sem. Mat. Univ. Padova. - 1980. - 62. - P. 207-219.

6. Ilolov M., Kuchakshoev Kh., Puchkov V., Yormamadov Sh. Equation of anomalous diffusion of cosmic rays // 34th Int. Cosmic Ray Conf. (Hague Netherlands, 30 July — 6 August, 2015). — P. 1-7.

7. Jun-Min Wang, Bao-Zhu Guo, Meng-Yin Fu Dynamic behavior of a heat equation with memory// Math. Meth. Appl. Sci. - 2009. - 32. - P. 1287-1310.

8. Grimmer R. Resolvent operators for integral equations in a Banach space// Trans. Am. Math. Soc. 1982. - 273. - P. 333-349.

9. Grimmer R., Pruss J. On linear Volterra equations in Banach spaces// Comput. Math. Appl. — 1983. — 11, № 1-3. - P. 189-205.

10. Hong-Ming Yin The classical solutions for nonlinear integro-differential equations// J. Integral Eqs. Appl. - 1988. - 1, № 2. - P. 249-262.

Илолов Мамадшо Илолович

Академия наук Республики Таджикистан, Душанбе, Таджикистан

E-mail: ilolov.mamadsho@gmail.com 